\title{
Mathematical Modeling of Aircraft Flying Qualities with Varying Tail Dihedral
}

\author{
M. Uzair Khan, Naveed A. Din, M. Diyan Khan, and M. Fawad Hussain
}

\begin{abstract}
V-tail is a tail geometry setup that provides stability and controllability about longitudinal and directional axes simultaneously. In addition, the setup has less wetted area and interference, thus producing less drag as compared to conventional tails. The dihedral angle of a V-tail determines its contribution to both longitudinal and directional dynamics. However, there is no well-defined empirical method to compute the most suitable dihedral angle for a V-tail in order to meet the required flying qualities. This work presents a method to select the most appropriate dihedral angle of a V-tail to fulfill the requirements of aircraft flying qualities. Numerical calculations were used to generate a complete flight dynamics model with different tail dihedral angles. Subsequently, damping ratios for longitudinal and lateral-directional modes were extracted from these models. Using a curve fitting technique a polynomial was generated for longitudinal and lateral-directional damping ratios against tail dihedral angle. It was observed that by increasing the tail dihedral the longitudinal damping ratio was reduced. In addition, the lateral-directional damping ratio increased with the increase in tail dihedral angle. The lower bound of the tail dihedral angle was obtained using the lateral-directional damping limit in accordance to the flying qualities. Similarly, the upper bound of the tail dihedral angle was obtained using the longitudinal damping limit. The tail dihedral angle in between these bounds was found to be optimal for adequate longitudinal and lateraldirectional flying qualities. In addition, it was observed that the mathematical model was not valid for a different flight dynamics model. This is due to the change in aerodynamic behavior of the aircraft.
\end{abstract}

Index Terms-Athena VLM, curve fitting, damping ratio, flight dynamics, flying qualities, mathematical modelling, tail dihedral, vortex lattice, v-tail.

\section{INTRODUCTION}

Aircraft tail geometry can significantly impact its stability and controllability. Its influence on flight dynamics depends on a number of geometrical parameters such as planform area, longitudinal location, sweep angle and tail dihedral. These parameters also effect the aerodynamic performance of an aircraft. By increasing the planform area one can achieve better stability and controllability characteristics however, also compromise on the aerodynamic efficiency of the aircraft due to the increasing drag [1]. Conventional Ttail setup as shown in Fig. 1(a) consists of a horizontal

Manuscript received June 29, 2019; revised August 6, 2019.

M Uzair Khan, Naveed Akmal Din are with Mechanical Engineering Department EME. National University of Sciences and Technology, Islamabad, Pakistan (e-mail: uzair01994@gmail.com, naveeddin@ceme.nust.edu.pk).

M Diyan Khan and M Fawad Hussain are with Flight Controls Team, AvRID, Pakistan Aeronautical Complex, Kamra, Pakistan (e-mail: diyankhan@aerospace.pk, hussain.fawad@gmail.com). stabilizer and a vertical stabilizer. These stabilizers have their corresponding drag contribution due to the respective planform areas. An additional drag force is produced due to the aerodynamic interference in between these stabilizers. Therefore, the drag penalty of a conventional tail setup is significant [2].

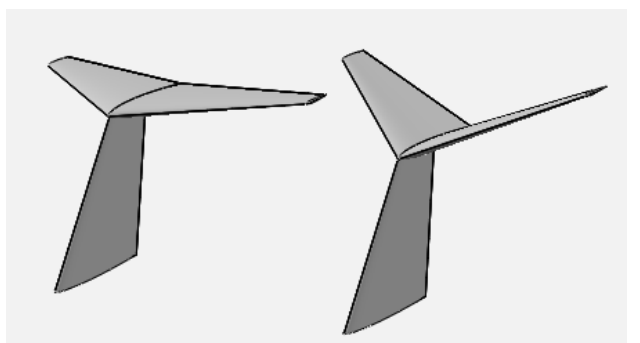

Fig. 1. (a) Left: Conventional T-tail setup. (b) Right: Conventional T-tail setup with horizontal tail dihedral.

In order to increase the positive tail contribution to stability and controllability, a certain geometrical change is required which also improves aerodynamic efficiency. Tail dihedral is a geometrical parameter which can influence aircraft stability. By increasing tail dihedral aircraft directional static stability increases due to an addition in vertical projection area of the tail. On the other hand, increasing tail dihedral reduces longitudinal static stability due to reduction in horizontal projection area of the tail [3]

Dihedral angle of the horizontal tail can be selected in such a way to provide adequate static longitudinal stability and directional stability. A horizontal tail with dihedral angle provides better stability and control characteristics if it is mounted above the vertical tail. This particular tail setup produces less interference drag as compared to a conventional T-tail setup [4].

However, by increasing the horizontal tail dihedral angle, mounted at the root of the vertical stabilizer, causes the interference drag to increase due to reduced gap and increased vorticity between the respective surfaces especially during a combined angle of attack and sideslip motion [5].

The dihedral angle also reduces the need of a large vertical stabilizer thus, reducing the drag contribution of the vertical stabilizer. Therefore, a horizontal tail dihedral (VTail) can provide directional and longitudinal stability and control simultaneously with the advantage of producing less drag as compared to conventional T-tail setup [6].

The dihedral angle of the horizontal tail reduces static longitudinal stability and increases static directional stability. Thus, increasing the dihedral angle has its advantages as well as disadvantages. Therefore, it is a necessity to select the most optimal dihedral angle in tail design procedure in order to prevent the loss of static longitudinal stability below 
standard requirements.

It is essential for the aircraft tail setup (with or without dihedral angle) to provide sufficient static longitudinal and directional stability in order to fulfill the requirements of aircraft flying qualities (MIL-F-8785C) mentioned in Table I. These requirements are of longitudinal and lateraldirectional dynamics in terms of their respective damping ratios.

TABLE I: AIRCRAFT FLYING QUALITIES (MIL-F-8785C) [7]

\begin{tabular}{lll}
\hline \hline & $\begin{array}{l}\text { Longitudinal Damping } \\
\text { Ratio }\end{array}$ & $\begin{array}{l}\text { Lateral-Directional } \\
\text { Damping Ratio }\end{array}$ \\
\hline Level 1 & 0.35 & 0.08 \\
Level 2 & 0.25 & 0.02 \\
Level 3 & 0.15 & 0.00 \\
\hline \hline
\end{tabular}

This research work presents a method to select the most appropriate dihedral angle of horizontal tail to provide sufficient longitudinal and lateral-directional damping ratio. The method is based on mathematically modelling the change in longitudinal damping ratio and lateral-directional damping ratio with change in horizontal tail dihedral angle. The tail setup used in this research is a T-tail setup as the dihedral angle in this particular tail setup provides positive effects to aircraft aerodynamics (increased stability and reduced drag).

\section{Methodology}

An aircraft geometry is required to perform stability and control analysis. A high subsonic speed executive jet was used for this purpose. Using the available geometric parameters a 3D CAD model was generated. The CAD model was altered by changing the tail dihedral angle as given in Table II and multiple CAD models were generated with different dihedral angles of horizontal tails.

\begin{tabular}{|c|c|}
\hline \multicolumn{2}{|c|}{ DIHEDRAL ANGLES } \\
\hline Configuration & Tail Dihedral (degrees) \\
\hline 1 & 0 \\
\hline 2 & 10 \\
\hline 3 & 20 \\
\hline 4 & 30 \\
\hline 5 & 40 \\
\hline
\end{tabular}

These geometries were imported into an extended Vortex lattice Method (VLM) environment defined in [8]. Aerodynamic analysis via VLM was performed at 0.8 Mach and at an altitude of 41000 feet. Stability derivatives obtained from this particular aerodynamic analysis, for different tail configurations were recorded and analyzed.

These derivatives were plugged in state matrices to form a complete state space system and its corresponding eigen values. The eigen values for longitudinal and lateraldirectional dynamics were distinguished and were used to compute the damping ratio of respective dynamic modes as done in [9].
The damping ratios for longitudinal and lateral-directional motion against different tail configurations were compared. Plots were generated for different longitudinal and lateraldirectional damping ratios against all tail configurations. This data obtained from preliminary analysis was used to obtain a relation between the damping ratio of longitudinal and lateral-directional modes with tail dihedral angle. This relation was obtained using curve fitting technique in MATLAB.

Using aircraft handling qualities mentioned in Table I, an acceptable range of damping ratio for longitudinal and lateral-directional motion was obtained for Level 1 flying quality. This particular range of longitudinal and lateraldirectional damping ratio was mapped onto the mathematical model between tail dihedral angle and damping ratio to obtain the lower and upper bound for the tail dihedral angle. Any value of tail dihedral angle in between these bounds would provide sufficient longitudinal and directional stability to meet the aircraft flying qualities.

Fig. 2 represents a schematic flow diagram of the methodology used.

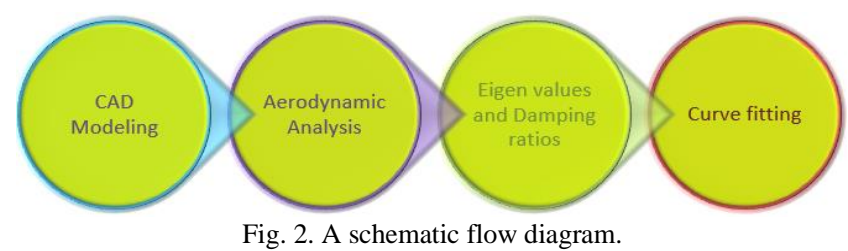

\section{IMPLEMENTATION}

For aerodynamic analysis Athena Vortex Lattice Method (AVL) was used. AVL is an extended vortex lattice method (VLM) software that supports aircraft configuration development by offering aerodynamic analysis, trim calculation, static and dynamic stability analysis. AVL is a program for the aerodynamic and flight-dynamic analysis of rigid aircraft of arbitrary concept. It is based on Prandtl's classic lifting line theory as defined in [10]. It employs an extended vortex lattice model for the lifting surfaces, together with a slender-body model for fuselages and nacelles. The flight dynamic analysis combines a full linearization of the aerodynamic model about any flight state, together with specified mass properties.

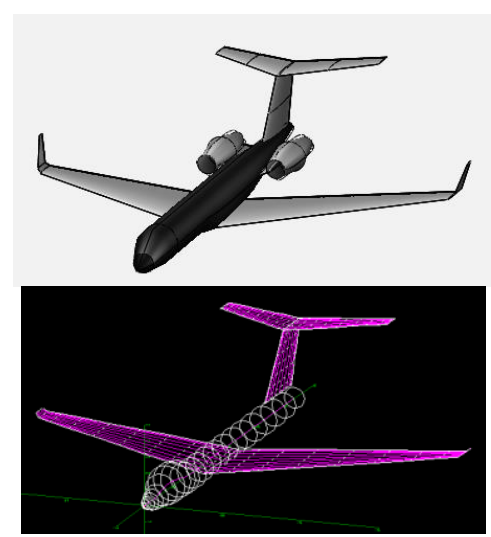

Fig. 3. The 3D CAD model of the executive jet (above). Geometry generated by AVL for the executive jet using panel distribution (below).

The geometry imported into AVL consists of a wing with 
zero dihedral and no twist. Therefore, no correction for wing twist was required to execute the lifting line analysis in extended vortex lattice method to compute the aerodynamic coefficients [11], [12].

Geometrical parameters of a high subsonic executive jet were imported into AVL environment using a series of input files. The geometry thus generated is shown in Fig. 3. The mass properties and flight conditions were also used as inputs. Cruise flight condition was used at $0.8 \mathrm{Mach}$ and at an altitude of 41000 feet.

Fig. 4 shows the geometry of Gulfstream G-550 with tail dihedral angle of 30 degrees.

Aerodynamic and stability coefficients obtained from AVL were recorded. These coefficients were recorded for all tail configurations mentioned in Table II.

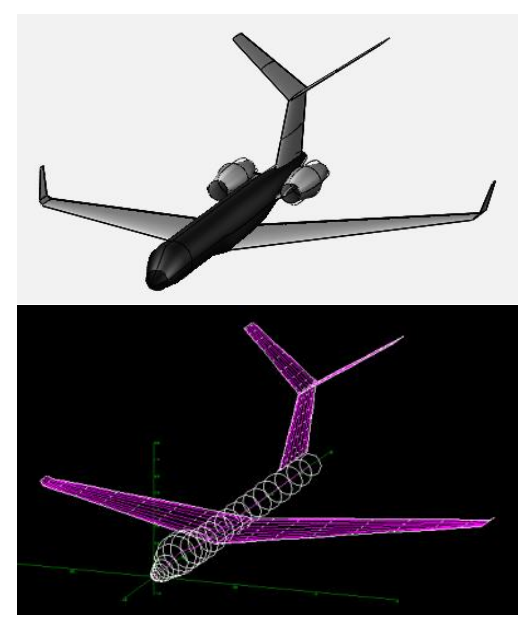

Fig. 4. 3D CAD model (above) and VLM panels generated by AVL (below) for tail dihedral angle of 30 degrees.

The coefficients were plugged in state space matrices to form a complete state space system. The state space system captured both longitudinal and lateral-directional dynamics of the aircraft. Thus, the matrices were used to compute longitudinal and lateral-directional eigen values using MATLAB. Subsequently, these eigen values were used to compute damping ratio and of the respective dynamic modes using equation (1) and (2).

$$
\begin{gathered}
\lambda=\eta \pm i \omega \\
\zeta=\cos \left(\tan ^{-1}\left(\frac{\omega}{\eta}\right)\right)
\end{gathered}
$$

where, $\eta$ is the real part of the eigen value and $\omega$ is the imaginary part of the eigen value [1]. The longitudinal and lateral-directional damping ratios were recorded against each tail dihedral configuration. The data generated was transformed into mathematical equations, for longitudinal and lateral-directional damping ratios, using curve fitting technique in MATLAB.

For the most accurate curve fitting, different techniques were used to generate appropriate polynomial curves of different degrees. The $R^{2}$ value was calculated for each curve fitted onto the data. This value for each polynomial fit was compared.

Table III shows minimal error when $3^{\text {rd }}$ degree (or higher) polynomial is selected for curve fitting. Therefore, a $3^{\text {rd }}$ order polynomial was selected for curve fitting technique on longitudinal and lateral-directional damping ratios.

TABLE III: COMPARISON OF THE R SQUARE VALUE FOR DIFFERENT POLYNOMIALS USED FOR CURVE FITTING FOR LONGITUDINAL AND LATERAL-DIRECTIONAL DAMPING RATIOS

\begin{tabular}{lll}
\hline \hline Polynomial & $R^{2}\left(\zeta_{\text {LONG }}\right)$ & $R^{2}\left(\zeta_{\text {LAT }}\right)$ \\
\hline Linear & 0.8986 & 0.9395 \\
$2^{\text {nd }}$ Degree & 0.9574 & 0.9969 \\
$3^{\text {rd }}$ Degree & 0.9684 & 0.9998 \\
$4^{\text {th }}$ Degree & 0.9998 & 0.9998 \\
\hline \hline
\end{tabular}

After generating two different mathematical relations between longitudinal damping ratio, lateral-directional damping ratio and tail dihedral angle, the lower and upper bound of damping ratio was selected in accordance to the flying qualities in Table I. From these limits of damping ratios, the lower and upper bound of the tail dihedral angle was determined. Any tail dihedral angle beyond these limits would result in an unacceptable flying quality in either longitudinal or lateral-directional motion.
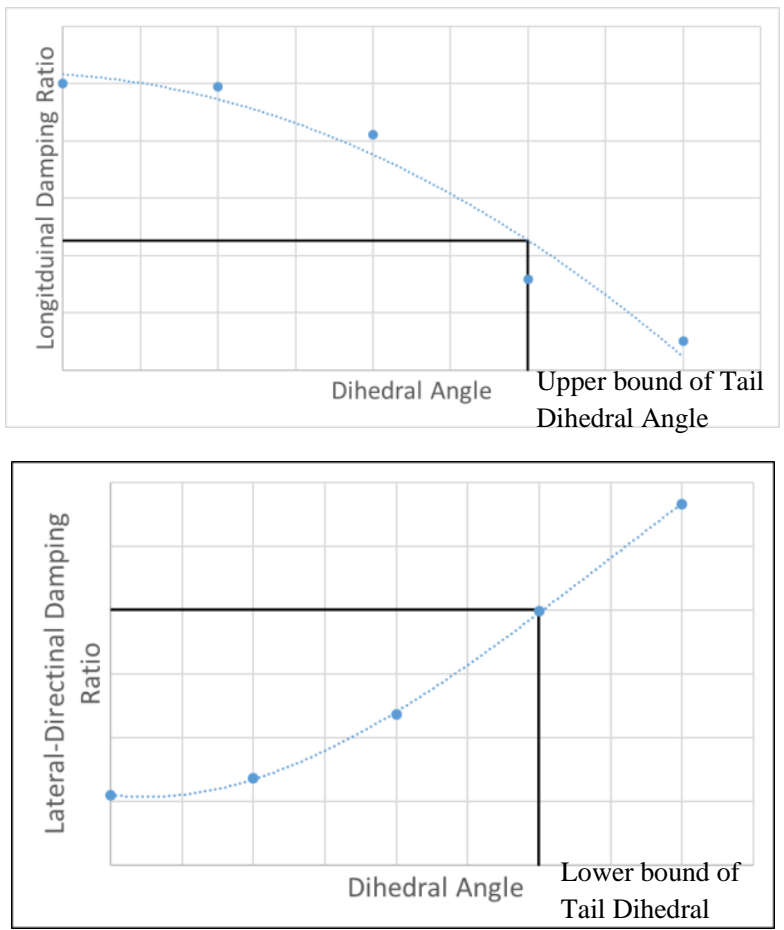

Fig. 5. A schematic graphical representation of the upper bound of tail dihedral angle from longitudinal damping ratio limit (above) and lower bound of tail dihedral angle from lateral-directional damping ratio limit (below).

It can be observed from Fig. 5 that the lower bound of dihedral angle is governed by lateral-directional damping ratio limit. This is due to the reason that by decreasing the tail dihedral angle the side projected area of the tail reduces, thus reducing the directional stability and the lateraldirectional damping ratio. Similarly, the upper bound of dihedral angle is governed by longitudinal damping ratio limit as by increasing the dihedral angle of the tail reduces the horizontal projection area of the tail and thus, reduced longitudinal stability and damping ratio.

The tail dihedral angle should be of any value in between the lower and the upper bounds. These bounds are governed by the aircraft flying qualities and would always ensure 
adequate longitudinal and lateral-directional dynamic stability to meet the flying quality requirements.

\section{RESUlTS AND DisCUSSION}

The impact of changing tail dihedral was recorded in terms of changes in longitudinal and directional static stability. In addition, the change in dynamic response (damping ratio) of the aircraft about longitudinal and lateraldirectional axes was also recorded.
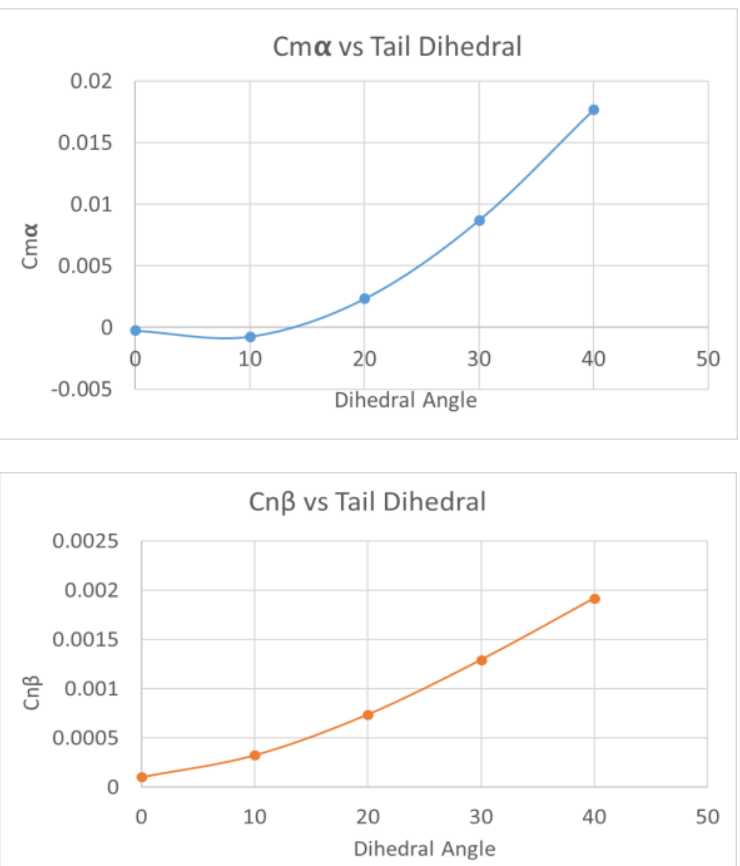

Fig. 6. Plots for change in $\mathrm{Cm} \alpha$ (above) and $\mathrm{Cn} \beta$ (below) derivatives with respect to tail dihedral angle.

Fig. 6 shows the change in static longitudinal and directional stability with change in tail dihedral angle. It can be observed that the static directional stability increases with increase in tail dihedral angle.

This is due to increase in the vertical area projection of the tail. On the other hand, static longitudinal stability decreases with increase in tail dihedral angle due to decrease in horizontal projection area of the tail. This reduction in horizontal projection area reduces the lifting force and consequently reduces the pitching moment.

TABLE IV: CHANGE IN COEFFICIENT OF LIFT AND COEFFICIENT OF Pitching Moment, at Zero ANGLE of ATtack, With TAil DiHedRAL

\begin{tabular}{lll}
\multicolumn{3}{c}{ ANGLE } \\
$\begin{array}{l}\text { Tail Dihedral } \\
\text { (Degrees) }\end{array}$ & $\mathrm{Cm}$ & $\mathrm{CL}$ \\
\hline 0 & 0.0828 & -0.0156 \\
10 & 0.0756 & -0.0144 \\
20 & 0.0658 & -0.0126 \\
30 & 0.0544 & -0.0104 \\
40 & 0.0426 & -0.0082 \\
\hline \hline
\end{tabular}

Change in static longitudinal stability for tail dihedral angle of 10 degrees is minimum due to a very small change in lifting force and pitching moment generated by the tail.
Table IV records the coefficients of the lifting force and pitching moment generated by the tail at zero angle of attack. It can be observed that the change in these coefficients also increase with the increase in tail dihedral angle.
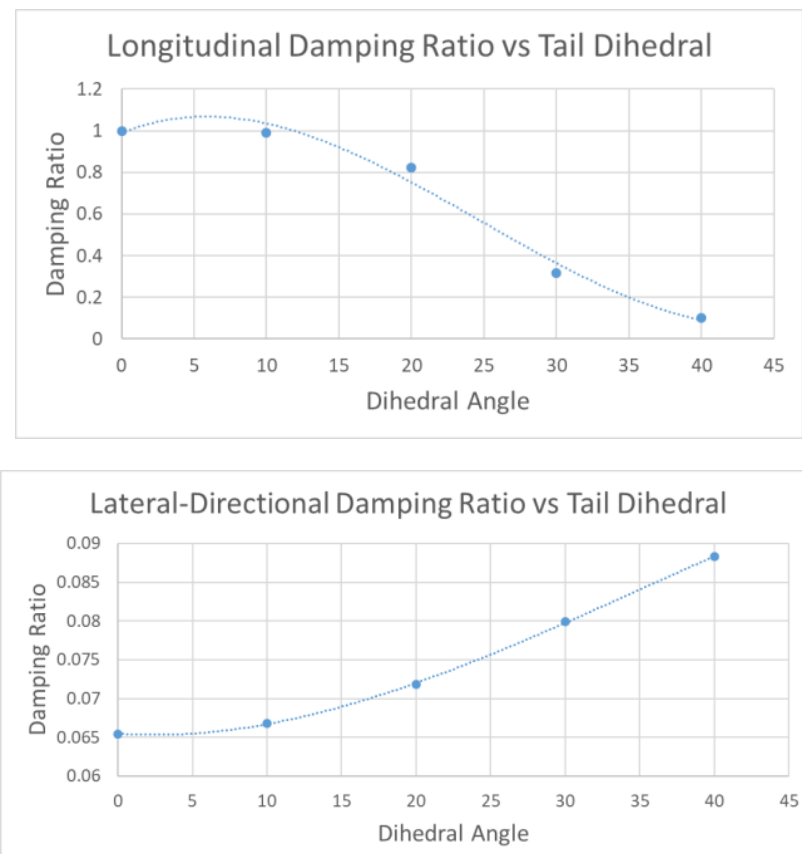

Fig. 7. Change in longitudinal (above) and lateral-directional (below) damping ratios with tail dihedral angle.

Dynamic stability is represented in the form of different dynamic modes for longitudinal and lateral-directional axes. The damping ratios corresponding to these modes were recorded and plotted against tail dihedral angle.

Fig. 7 shows change in longitudinal and lateral-directional damping ratios with tail dihedral angle. It can be observed that longitudinal damping ratio decreases by increasing tail dihedral angle whereas lateral-directional damping ratio increases by increasing tail dihedral angle. The increment of lateral-directional damping ratio is due to the increase in vertical projection area of the tail (with increasing tail dihedral angle) and thus, increase in the directional stability of the aircraft. The decrement of longitudinal damping ratio is due to the decrease in horizontal projection area with increasing tail dihedral angle.

The data obtained for longitudinal and lateral-directional damping ratio was used to predict a suitable equation via curve fitting method. A $3^{\text {rd }}$ order polynomial was generated with a $R^{2}$ value recorded in Table III. The mathematical equations for longitudinal and lateral-directional damping ratios computed using curve fitting techniques are as follows:

$$
\begin{gathered}
\zeta_{\mathrm{LONG}}=4 \times 10^{-5} \Gamma^{3}-0.0028 \Gamma^{2}+0.0284 \Gamma+0.9885 \\
\zeta_{\mathrm{LAT}}=-3 \times 10^{-7} \Gamma^{3}+3 \times 10^{-5} \Gamma^{2}-0.0001 \Gamma+0.0655
\end{gathered}
$$

where $\zeta_{\mathrm{LONG}}$ and $\zeta_{\mathrm{LAT}}$ are the longitudinal and lateraldirectional damping ratios respectively. $\Gamma$ is the tail dihedral angle.

Using international standards in [7] which define handling qualities of an aircraft, damping ratio values for level 1 flying qualities were recorded for both longitudinal and lateral-directional modes. These values were then 
incorporated in equation (3) and (4) to calculate upper and lower bounds for tail dihedral angle. As mentioned earlier lower bound for tail dihedral angle was governed by lateraldirectional damping ratio limit while the upper bound for tail dihedral angle was governed by the longitudinal damping ratio limit. The tail dihedral angles found using these relations are as follows:

TABLE V: UPPER AND LOWER BOUNDS OF TAIL DIHEDRAL ANGLE OBTAINED USING MATHEMATICAL MODEL GENERATED

\begin{tabular}{ll}
\hline \hline & Dihedral angle (degrees) \\
\hline Upper bound & 31.6751 \\
Lower bound & 28.4172 \\
\hline \hline
\end{tabular}

Table $\mathrm{V}$ records the upper and lower bounds of tail dihedral angle that ensures Level 1 flying qualities of longitudinal and lateral-directional dynamics. The tail dihedral angle should lie within these bounds.

Aerodynamics of the aircraft changes with change in flight speed and Mach number. This change also effects the flight dynamics and subsequently the longitudinal and lateral-directional damping ratios. In order to test the mathematical model obtained at high subsonic Mach number of 0.8 , numerical analysis was performed for the same executive jet at low subsonic Mach numbers of 0.3 and 0.5 with different tail dihedral angles. The damping ratios obtained at these two subsonic Mach numbers were recorded and compared with the damping ratios obtained using the mathematical model.

TABLE VI: LONGITUDINAL AND LATERAL-DIRECTIONAL DAMPING RATIOS RECORDED AND LOW SUBSONIC MACH NUMBERS FOR THE SAME

\begin{tabular}{|c|c|c|c|c|}
\hline $\begin{array}{l}\text { Mach } \\
\text { No. }\end{array}$ & $\begin{array}{l}\text { Dihedral } \\
\text { (degrees) }\end{array}$ & Axis & $\begin{array}{c}\zeta \\
\text { (Numerical) }\end{array}$ & $\begin{array}{c}\zeta \\
\text { (Model) }\end{array}$ \\
\hline \multirow{4}{*}{0.3} & \multirow{2}{*}{10} & Longitudinal & 0.6899 & 0.9925 \\
\hline & & $\begin{array}{l}\text { Lateral- } \\
\text { Directional }\end{array}$ & -0.0761 & 0.0672 \\
\hline & \multirow{2}{*}{20} & Longitudinal & 0.8187 & 0.7565 \\
\hline & & $\begin{array}{l}\text { Lateral- } \\
\text { Directional }\end{array}$ & -0.0542 & 0.0731 \\
\hline \multirow{4}{*}{0.5} & \multirow{2}{*}{10} & Longitudinal & 0.7486 & 0.9925 \\
\hline & & $\begin{array}{l}\text { Lateral- } \\
\text { Directional }\end{array}$ & 0.0048 & 0.0672 \\
\hline & \multirow{2}{*}{20} & Longitudinal & 0.9398 & 0.7565 \\
\hline & & $\begin{array}{l}\text { Lateral- } \\
\text { Directional }\end{array}$ & 0.0204 & 0.0731 \\
\hline
\end{tabular}

It can be observed from Table VI that the same mathematical model generated at a high subsonic Mach number cannot be used for predicting damping ratios at low subsonic Mach numbers. At low subsonic Mach numbers there is a considerable difference between the longitudinal damping ratios obtained using the mathematical model and Athena VLM (numerical). In addition, the lateral-directional dynamics of the aircraft completely change at low subsonic Mach numbers, as presented in the table above and cannot be predicted using the mathematical model at high subsonic Mach numbers. The difference is due to a complete shift in aerodynamics of the aircraft. With change in Mach number the complete longitudinal and lateral-directional dynamics of the aircraft changes.

TABLE VII: ChANGE IN AERODYNAMC COEFFICIENTS With CHANGE IN FLIGHT MACH NUMBER

\begin{tabular}{lllll}
\hline \hline $\begin{array}{l}\text { Mach } \\
\text { No. }\end{array}$ & $\begin{array}{l}C_{\mathrm{m} \alpha} \\
\text { (per radian) }\end{array}$ & $\begin{array}{l}C_{\mathrm{n} \beta} \\
(\text { per radian) }\end{array}$ & $\begin{array}{l}C_{\mathrm{l} \beta} \\
(\text { per radian) }\end{array}$ & $X_{\mathrm{N} . \mathrm{P}}$ \\
\hline 0.3 & -0.2701 & 0.0332 & -0.0435 & 15.84 \\
0.5 & -0.2316 & 0.0310 & -0.0456 & 15.82 \\
0.8 & -0.0411 & 0.0187 & -0.0531 & 15.75 \\
\hline \hline
\end{tabular}

From Table VII it can be observed that there is a significant change in the values of $C_{\mathrm{m} \alpha}, C_{\mathrm{n} \beta}$ and $C_{\mathrm{l} \beta}$ from subsonic Mach number of 0.5 to 0.8 . However, there is a very small change in these coefficients from Mach 0.3 to 0.5 . Therefore, there is significant change in aircraft dynamics (damping ratios) as the aerodynamic behavior of the aircraft completely changes. As a result, the mathematical model can only be used to predict the damping ratios and subsequently the most suitable tail dihedral angle for a single aerodynamic model.

\section{CONCLUSION}

A mathematical model was generated relating tail dihedral angle and damping ratios of longituduinal and lateral-directional dynamic modes. An executive jet was selected and anaylzed at high subsonic cruise flight condition. A trend was generated between the tail dihedral angle and the damping ratios of longitudinal and lateraldirectional modes. It was observed that longitudinal damping ratio decreased with increasing tail dihedral angle and vice versa for lateral-directional damping ratio. The data of damping ratios generated at different tail dihedral angles was used to formulate the mathematical model for longitudinal and lateral-driectional damping ratios. This model was used to determine a range of tail dihedral angles in order to fulfill the criteria of acceptable aircraft flying qualities.

This particular technique can be used in the early conceptual design phase to determine the effects of tail dihedral angle on dynamic stability of the aircraft.

Furthermore, the mathematical model was compared against numerical analysis on low subsonic Mach numbers. It was observed that the mathematical model was not able to predict longitudinal and lateral-directional damping ratio correctly at low subsonic Mach numbers. This is due to the change in aerodynamic behavior of the aircraft as Mach number changes. Thus, the mathematical model was found to be invalid for a different aerodynamic behavior. Therefore, it can also be concluded that the mathematical model is invalid for a completely different aircraft at a high subsonic Mach number.

A further breakdown analysis of the generated mathematical model is recommended. Each coefficient in the equations of longitudinal and lateral-directional damping ratios (equations (3) and (4) depends on specific parameters (aerodynamic, mass or geometrical). Therefore, it is necessary to find out those parameters that govern the values of these coefficients and thus the complete mathematical model. 


\section{REFERENCES}

[1] D. R. C. Nelson, Flight Stability and Automatic Control, R R donnelley and Sons Company.

[2] N. T. Dat, T. N. Son, and D. A. Tra, "Development of a Flight Dynamics model for fixed wing aircraft," ICCMS, 2018.

[3] J. A. Kidd, "Investigation of the effect of variable tail dihedral on airplane stability and control," AIAA, Aug. 1998.

[4] W. F. Phillips, A. B. Hansen, and W. M. Nelson, "Effects of tail dihedral on static stability," Journal of Aircraft, vol. 43, pp. 18291837, 2006.

[5] M. J. Abzug, "V-tail stalling at combined angles of attack and sideslip," Journal of Aircraft, vol. 36, 1999.

[6] P. E. Purser and J. P. Campbell, Experimental Verification of a Simplified Vee-Tail Theory and Analysis of Available Data on Complete Models with Vee Tails, NACA TR-823.

[7] D. J. Roskam, Airplane Design Part-1, 1995.

[8] M. Drela and H. Youngren, AVl doc, MIT Aero \& Astro Harold Youngren, Aerocraft, Inc., February 12, 2017.

[9] S. Swankar, H. Parwana, M. Kothari, and Abishek, "Development of flight dynamics model and control of Biplane-quadrotor UAV," in Proc. AIAA Guidance, Navigation and Control Conference, Kissimmee, Florida, 12 January 2018.

[10] W. F. Phillips and D. O. Synder, "Modern adaptation of prandtl's classic lifting-line theory," Journal of Aircraft, AIAA, pp. 662-670, 2000 .

[11] W. F. Phillips, N. R. Alley, and W. D. Goodrich, "Lifting-line analysis of roll control and variable twist," Journal of Aircraft, vol. 41 pp. 1169-1176, 2004.

[12] W. F. Phillips, "Lifting-line analysis for twisted wings and washoutoptimized wings," Journal of Aircraft, vol. 41, pp. 128-136, 2004.

Copyright (C) 2019 by the authors. This is an open access article distributed under the Creative Commons Attribution License which permits unrestricted use, distribution, and reproduction in any medium, provided the original work is properly cited (CC BY 4.0).

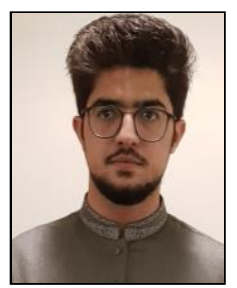

M. Uzair Khan completed his masters of science in mechanical engineering from National University of Sciences and Technology (NUST), Pakistan in August 2019 and his Bachelors of science from Heavy Industries Taxila Education City (HITEC), Pakistan in September 2016.

He works as a design engineer at AvRID Section, Pakistan Aeronautical Complex (PAC), Kamra, Pakistan since October 2017. He is currently working on flight dynamics modeling of aircraft.
Mr. Khan is currently a member of Pakistan Engineering Council (PEC) since 2017. He was awarded Silver Medal during his bachelor degree by the Mechanical Department of HITEC.

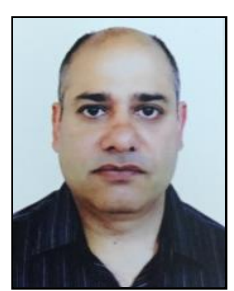

Naveed A. Din completed his postgraduate doctoral degree in mechanical engineering from University of Manchester, UK in September 2015.

$\mathrm{He}$ works as an assistant professor at National University of Sciences and Technology (NUST, Pakistan since October 2016. He is currently teaching "Advanced Theory of Vibrations" at NUST. His principal research activities have evolved from his $\mathrm{PhD}$ work about plate and beam vibrations and centre on the engineering applications of viscoelastic and smart materials for vibration and shock isolation and control.

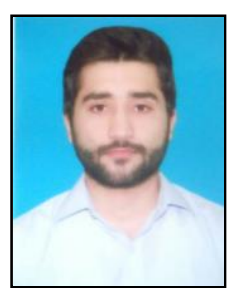

M. Diyan Khan completed his bachelors of engineering in aerospace engineering from National University of Sciences and Technology (NUST), Pakistan in September 2017.

He works as a design engineer at AvRID Section, Pakistan Aeronautical Complex (PAC), Kamra, Pakistan since October 2017. He is currently working as a member of Flight Controls Group at AvRID, PAC Kamra. He published a research article in 5th International Conference on Aerospace Science and Engineering, Islamabad with the title of "Stability Improvements of a novel Pigeon shaped Micro Air Vehicle".

Mr. Khan is currently a member of Pakistan Engineering Council (PEC) since 2018. He was awarded with Rector's Gold Medal for Best Final Year Project during his bachelor degree by the Aerospace Engineering Department of NUST.

M. Fawad Hussain completed his bachelors of engineering in avionics engineering from National University of Sciences and Technology (NUST) Pakistan in November 2007. He is currently pursuing masters of science in RF and Microwave from Air University, Pakistan.

He works as a deputy director of Fight Controls team at AvRID Section, Pakistan Aeronautical Complex (PAC), Kamra, Pakistan since October 2017. He published a research article in Wiley Periodicals, Inc. Microwave Opt Tech Letters with the title of "An Ultra Wideband Inverted Double Discone Antenna with 150:1 Impedance Bandwidth" in 2016.

Mr. Hussain is currently a member at Royal Aeronautical Society (RAeS) since 2018. He was awarded with Sword of Honor for best overall performance by Pakistan Air Force during his bachelor degree. 